Review

\title{
Immune-Checkpoint Inhibitors as the First Line Treatment of Advanced Non-Small Cell Lung Cancer: A Meta-Analysis of Randomized Controlled Trials
}

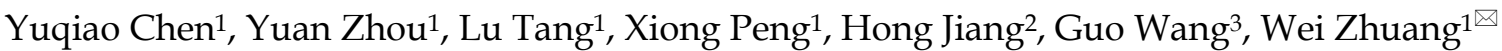 \\ 1. Department of Thoracic Surgery, Xiangya Hospital of Central South University, 410008 Changsha, Hunan, People's Republic of China. \\ 2. Department of Neurology, Xiangya Hospital, Central South University, 410008, Changsha, Hunan, People's Republic of China. \\ 3. Department of Clinical Pharmacology, Xiangya Hospital, Central South University, Changsha 410008, Hunan, People's Republic of China. \\ $\triangle$ Corresponding author: Wei Zhuang, Xiangya Hospital of Central South University, 410008 Changsha, Hunan, People's Republic of China. E-mail: \\ zhuangwei@csu.edu.cn. Tel: +86-152-0089-9869, Fax: +86-731-8432-7623 \\ (C) The author(s). This is an open access article distributed under the terms of the Creative Commons Attribution License (https://creativecommons.org/licenses/by/4.0/). \\ See http://ivyspring.com/terms for full terms and conditions.
}

Received: 2019.03.06; Accepted: 2019.08.26; Published: 2019.10.17

\begin{abstract}
Background: This meta-analysis aimed to explore if immunotherapy or chemotherapy alone or in combination is a better first line treatment strategy for advanced non-small cell lung cancer (NSCLC) patients.

Methods: Electronic databases including Google Scholar, PMC, PubMed, EMBASE, Scopus and the major conference proceedings were searched for relevant randomized controlled trials (RCTs) comparing outcomes of immune-checkpoint inhibitor combined with chemotherapy or immune-checkpoint inhibitor alone over chemotherapy alone in patients with advanced NSCLC without previous treatment. Study heterogeneity was assessed using the 12 test.

Results: A total of 14 RCTs including 8,081 treatment naive advanced NSCLC patients were enrolled in this study. Our results showed that in comparison to chemotherapy alone, introducing immunotherapy into first-line chemotherapy has significant benefit in tumor response (RR, 1.27; $95 \% \mathrm{Cl}, 1.09$ to 1.48 ), progression-free survival (PFS) (HR, $-0.43 ; 95 \% \mathrm{Cl},-0.56$ to -0.31 ), and overall survival (OS) (HR, $-0.30 ; 95 \% \mathrm{Cl},-0.45$ to -0.14 ) but with an increased risk of grade $3-5$ toxicity (RR, $1.11 ; 95 \% \mathrm{Cl}, 1.04$ to 1.18 ). The pooled results of comparison of immune therapy alone with chemotherapy alone in selected patients with positive expression of Programmed Death-ligament (PD-L1) or with a high tumor mutational burden, demonstrated similar tumor response (RR, 1.13; $95 \% \mathrm{Cl}, 0.88$ to 1.46$), 3-5$ grade toxicity $(\mathrm{RR}, 0.69 ; 95 \% \mathrm{Cl}, 0.40$ to 1.19$)$ and long-term outcomes, including OS (HR, $-0.20 ; 95 \% \mathrm{Cl},-0.43$ to 0.03 ) and PFS (HR, $-0.24 ; 95 \% \mathrm{Cl},-0.61$ to 0.14 ).

Conclusions: Our meta-analysis showed the superiority of combination therapy over monotherapy with chemotherapeutic agents in terms of tumor response, and long-term survival, but with an increased the $3-5$ grade toxicity. And immune-checkpoint inhibitors alone showed similar tumor response, toxicity and long-term outcomes compared to platinum-based chemotherapy in selected patients.
\end{abstract}

\section{Introduction}

Lung cancer is one of the most lethal diseases and has become the leading cause of cancer related deaths [1, 2]. Non-small cell lung cancer (NSCLC) is the largest subtype of lung cancer, comprising approximately $85 \%$ cases $[3,4]$. The first-line treatment strategy for advanced NSCLC is based on the expression of oncogenic aberrations, such as epidermal growth factor receptor gene (EGFR), anaplastic lymphoma kinase gene $(A L K)$, and orphan receptor tyrosine kinase (ROS) [1,5]. However, most 
patients with NSCLC do not harbor these genetic aberrations; thus, cytotoxic chemotherapy is still the first-line treatment for such patients [6, 7]. Chemotherapy alone is associated with a median overall survival (OS) of 8 - 10 months, progression-free survival (PFS) of 4 - 6 months, and objective tumor response rates of 25 - 35\% [8]. Moreover, the toxic effects of platinum-based chemotherapy are a concern for both clinicians and patients, as these severely impair quality of life [9]. Therefore, developing new agents with better effectiveness and less toxicity is crucial.

Drugs interrupting immune checkpoints, such as anti-cytotoxic T-lymphocyte-associated protein 4 (CTLA-4), anti-programmed cell death protein 1 (PD-1), anti-programmed cell death-ligand 1 (PD-L1), and others, can enhance anti-tumor immunity and mediate durable cancer regressions [10, 11]. Previous studies have demonstrated promising therapeutic value of immune checkpoint inhibitors as these lead to improved tumor responses, prolonged long-term survival, and less toxicity for patients with metastatic NSCLC who had progressed during or after platinum-based chemotherapy [11-16]. However, patients with advanced NSCLC usually undergo rapid deterioration during the first course of treatment, and less than half of these patients receive second-line therapy [17].

Over time several studies exploring the safety and efficacy of immunotherapy as the first-line treatment strategy for advanced NSCLC have been published [18-32]. Due to the availability of a wide range of immunotherapeutic agents and distinct treatment strategies, there is no unanimous conclusion about the therapeutic status of immunotherapy in the management of naïve NSCLC patients. Thus, we performed this meta-analysis of randomized controlled clinical trials (RCTs), which included patients with locally advanced NSCLC with metastasis, to ascertain whether immune checkpoint agents alone or in combination with chemotherapy improve survival outcomes in NSCLC patients who received chemotherapy alone as a first-line treatment.

\section{Material and methods}

\section{Study selection}

Electronic databases including Google Scholar, PMC, PubMed, EMBASE, Scopus, and the major conference proceedings (the American Society of Clinical Oncology and the European Society for Medical Oncology) were searched by two authors (Chen and Zhou) independently for RCTs published between $1^{\text {st }}$ January, 2010 and $1^{\text {st }}$ June, 2019. The following medical subject heading $(\mathrm{MeSH})$ terms were used: (1) "non-small cell lung cancer or NSCLC"; (2) "nivolumab or pembrolizumab or atezolizumab or ipilimumab or durvalumab"; (3) "PD-1 or PD-L1 or CTLA-4 or immune checkpoint inhibitor"; (4) "Randomized Controlled Trial or RCT". All potentially eligible and relevant clinical studies were manually retrieved and examined. Studies that met the following criteria were included in this meta-analysis: (a) RCTs; (b) studies comparing the combination of immune therapy and chemotherapy with chemotherapy alone in the treatment of advanced treatment-naive NSCLC patients; and (c) studies comparing immune therapy alone with chemotherapy alone in the treatment of advanced treatment-naive NSCLC patients. Non-randomized controlled trials, or studies unrelated to the first-line immune therapy, were excluded. Ultimately, 14 RCTs were included for quantitative analysis (Fig. 1). Any disagreements about the processes of study selection, data extraction, and methodological quality assessment were resolved by discussion and consensus with an independent expert (Zhuang).

\section{Data Extraction}

The following information from the eligible studies were extracted by two authors (Tang and Chen) independently: year of publication, number of included patients, treatment regimen, and clinical outcomes. Clinical outcome measures included tumor response, long-term survival [progression-free survival (PFS) and overall survival (OS)], and the toxicity (3-5 grade toxicity and toxicity leading to discontinuation of treatment). Tumor response was stratified as objective responders who obtained a complete or partial response and as non-responders who experienced a stable or progressive disease according to the Response Evaluation Criteria in Solid Tumors (RECIST) version 1.1 [33].

\section{Assessment of methodological quality}

Two authors (Chen and Zhou) independently assessed the methodological quality of the eligible studies according to the Cochrane Collaboration guidelines v5.1.0 [34].

\section{Data analysis}

All statistical analyses were performed using Stata12.0 software (Stata Corporation, College Station, TX, USA). Risk ratios (RRs) and hazard ratios (HRs) with 95\% confidence intervals (CIs) were calculated for dichotomous data. Heterogeneity among these included studies was evaluated using $\mathrm{I}^{2}$ statistics, where an $\mathrm{I}^{2}$ value $>50 \%$ was defined as substantial heterogeneity according to the Cochrane Collaboration guidelines v5.1.0 [34]. When $\mathrm{I}^{2}$ was < $50 \%$, the fixed-effects model was used to assess 
outcomes; otherwise, the random-effects model were preferred. Sensitivity analysis using both fixed and random-effect models for the same data was conducted to confirm the robustness and reliability of the results.

\section{Results}

\subsection{Search strategy}

The database search retrieved 5220 records. After deleting duplicate results, a total of 3410 abstracts were screened for eligibility, and 42 clinical trials were considered potentially eligible for inclusion based on titles and abstract review. After retrieving and further analyzing the full-text of these studies, another 28 studies were excluded; the remaining 14 studies [18-32] were finally included in the meta-analysis (Fig. $1)$. These 14 RCTs included a total of 8,081 patients: 4,391 patients had been administered immunotherapy alone or combination of chemotherapy and immunotherapy, and the remaining the 3,690 patients had been administered platinum-based chemotherapy alone. The characteristics of all 14 included studies are shown in Table 1. Among these 14 included studies, ten RCTs had compared combination of checkpoint inhibitor and chemotherapy with chemotherapy alone [18-26, 31, 32]. The other four RCTs had compared checkpoint inhibitor alone with chemotherapy alone [27-30].

\subsection{Outcome assessments}

\subsubsection{Tumor response}

The results based on ten RCTs [18-26, 31, 32] showed that combined immunotherapy and chemotherapy had significant benefit compared to chemotherapy alone with respect to tumor response (RR, 1.27; 95\% CI, 1.09 to 1.48 ; $\mathrm{I}^{2}=66.8 \%$ ) (Fig. 2). Additionally, the immune-checkpoint inhibitor alone was not inferior to chemotherapy alone as the first-line therapy with respect to tumor response rate (RR, 1.13; 95\% CI, 0.88 to 1.46; $\mathrm{I}^{2}=67.9 \%$ ) [27-30] (Fig. 2). As a whole, the use of the immunotherapy as the first-line therapy increased the objective tumor response (RR, 1.22; 95\% CI, 1.08 to $1.39 ; \mathrm{I}^{2}=65.7 \%$ ) (Fig. 2).

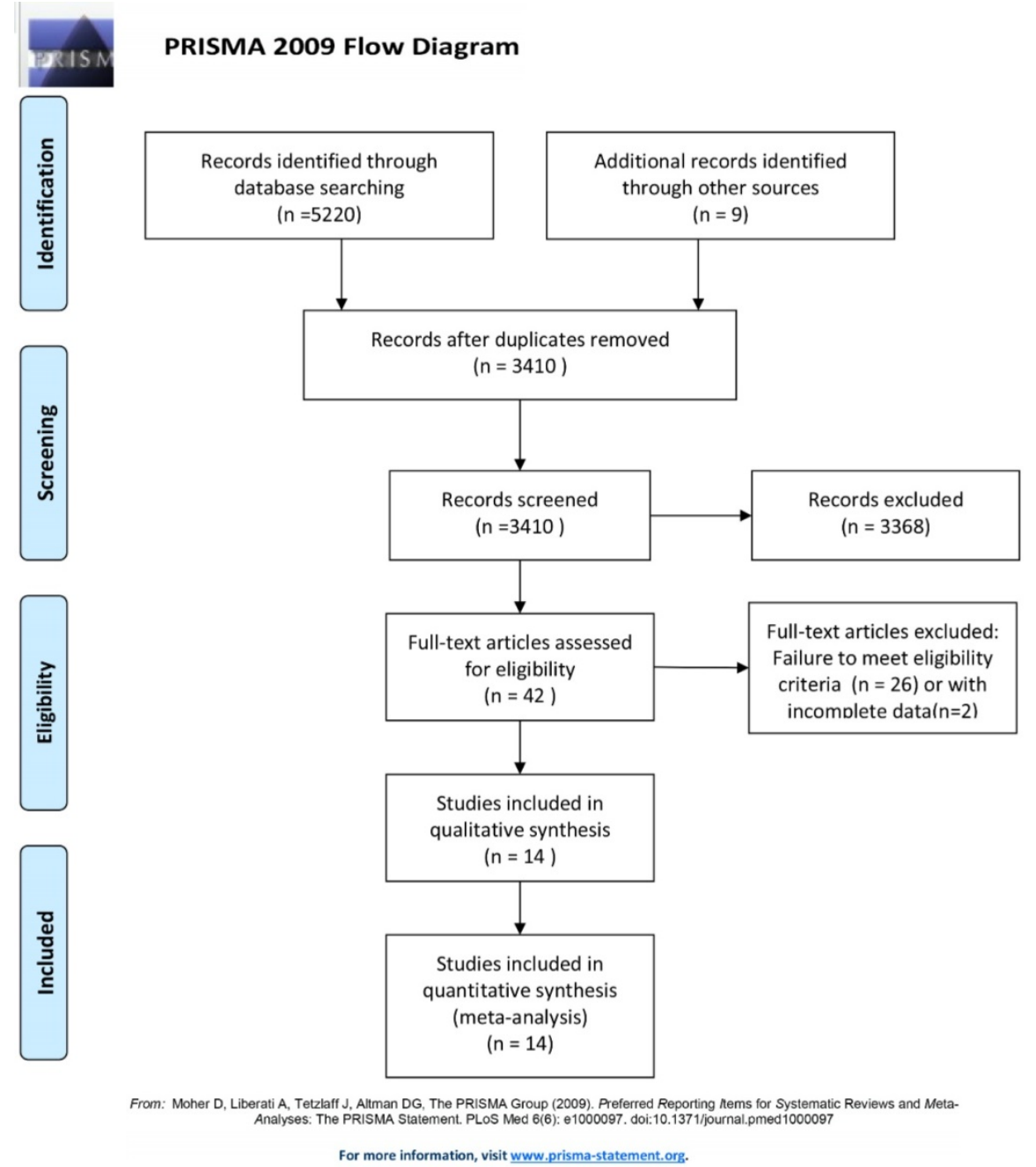

For more information, visit www. prisma-statement.org. 
Table 1. Summary of 14 randomized controlled trials included in the meta-analysis.

\begin{tabular}{|c|c|c|c|c|c|c|c|}
\hline \multirow{2}{*}{$\begin{array}{l}\text { Study } \\
\text { CA184-041 }\end{array}$} & \multirow{2}{*}{$\begin{array}{l}\text { Author } \\
\text { Lynch et al. [25] }\end{array}$} & \multirow{2}{*}{$\begin{array}{l}\text { Year } \\
2012\end{array}$} & \multicolumn{2}{|c|}{$\begin{array}{l}\text { Study Group } \\
\text { (regime and no. of Pts.) }\end{array}$} & \multicolumn{2}{|c|}{$\begin{array}{l}\text { Control Group } \\
\text { (regime and no. of } \\
\text { Pts.) }\end{array}$} & \multirow{2}{*}{$\begin{array}{l}\text { Inclusion criteria } \\
\text { Stage IIIB or IV NSCLC }\end{array}$} \\
\hline & & & Ipi+Chemo & 70 & Chemo alone & 66 & \\
\hline KEYNOTE 021 & Langer et al. [19] & 2016 & Pembro+Chemo & 60 & Chemo alone & 63 & $\begin{array}{l}\text { Stage IIIB or IV, non-squamous NSCLC without targetable genetic } \\
\text { aberration }\end{array}$ \\
\hline NCT01285609 & Govindan et al. [26] & 2017 & Ipi+Chemo & 388 & Chemo alone & 361 & stage IV or recurrent squamous NSCLC \\
\hline IMpower 150 & Socinski et al. [20] & 2018 & Atezo+Chemo & 400 & Chemo alone & 400 & $\begin{array}{l}\text { Stage IIIB or IV, non-squamous NSCLC without targetable genetic } \\
\text { aberration }\end{array}$ \\
\hline IMpower 131 & Jotte et al. [22] & 2018 & Atezo+Chemo & 343 & Chemo alone & 340 & Stage IV, squamous NSCLC without \\
\hline IMpower 132 & $\begin{array}{l}\text { Papadimitrakopoulou et } \\
\text { al. [23] }\end{array}$ & 2018 & Atezo+Chemo & 292 & Chemo alone & 286 & Stage IV non-squamous NSCLC without targetable genetic aberration \\
\hline KEYNOTE 407 & Paz-Ares et al. [21] & 2018 & Pembro+Chemo & 278 & Chemo alone & 281 & Stage IV, squamous NSCLC \\
\hline KEYNOTE 189 & Gandhi et al. [18] & 2018 & Pembro+Chemo & 410 & Chemo alone & 206 & Stage IV non-squamous NSCLC without targetable genetic aberration \\
\hline IMpower 130 & West et al. [24] & 2019 & Atezo+Chemo & 473 & Chemo alone & 232 & Stage IV, non-squamous NSCLC without targetable genetic aberration \\
\hline PACIFIC & Antonia et al. [31, 32] & 2018 & Durva+Chemo & 476 & Chemo alone & 237 & stage III, unresectable NSCLC \\
\hline KEYNOTE 024 & Reck et al. [29] & 2016 & Pembro alone & 154 & Chemo alone & 151 & $\begin{array}{l}\text { Stage IIIB or IV, NSCLC without targetable genetic aberration but with a } \\
\text { PD-L1 tumor-expression level of } 50 \% \text { or more }\end{array}$ \\
\hline CheckMate 026 & Carbone et al. [27] & 2017 & Nivo alone & 271 & Chemo alone & 270 & $\begin{array}{l}\text { Stage IV or recurrent NSCLC without targetable genetic aberration but with } \\
\text { a PD-L1 tumor-expression level of } 5 \% \text { or more }\end{array}$ \\
\hline CheckMate 227 & Hellmann et al. [28] & 2018 & Nivo+Ipi & 139 & Chemo alone & 160 & $\begin{array}{l}\text { Stage IV or recurrent NSCLC without targetable genetic aberration, with a } \\
\text { high tumor mutational burden ( } \geq 10 \text { mutations per megabase) }\end{array}$ \\
\hline KEYNOTE 042 & Mok et al. [30] & 2018 & Pembro alone & 637 & Chemo alone & 637 & $\begin{array}{l}\text { Stage IV or recurrent NSCLC without targetable genetic aberration but with } \\
\text { a PD-L1 tumor-expression level of } 1 \% \text { or more }\end{array}$ \\
\hline
\end{tabular}

Abbreviations: Pts -patients, Pembro- Pembrolizumab, Atezo-Atezolizumab, Nivo- Nivolumab, Ipi- Ipilimumab, Durva-Durvalumab, Chemo-Chemotherapy

\subsection{Toxicity}

The pooled results showed that the combination of immunotherapy and chemotherapy significantly increased toxicity compared to chemotherapy alone $\left(\mathrm{RR}, 1.11 ; 95 \% \mathrm{CI}, 1.04\right.$ to $\left.1.18 ; \mathrm{I}^{2}=7.2 \%\right)$ [18-26, 31, 32]. However, no significant difference in $3-5$ grade toxicity was found between patients in the monotherapy arms (RR, 0.69; 95\% CI, 0.40 to $1.19 ; \mathrm{I}^{2}=$ 94.2\%) (Fig. 3A) [27-30]. Furthermore, more patients who underwent the combination of immunotherapy and chemotherapy discontinued their treatment due to the toxicity in combination of immunotherapy and chemotherapy group compared to chemotherapy alone (RR, 1.46; 95\% CI, 1.23 to $1.74 ; \mathrm{I}^{2}=0 \%$ ) [18, 19, 21-23, 31, 32]. However, patients who discontinued their treatment due to toxicity was comparable between groups of immune therapy alone and chemotherapy alone (RR, 1.26; $95 \% \mathrm{CI}, 0.78$ to 2.04 ; $\mathrm{I}^{2}$ $=70.5 \%$ ) (Fig. 3B) [27-30].

\subsection{Progression-free survival and overall survival}

Based on random effects model analysis, a statistically significant benefit of combination of immune therapy and chemotherapy over chemotherapy alone was observed in term of PFS (HR, $-0.43 ; 95 \%$ CI, -0.56 to $-0.31 ; \mathrm{I}^{2}=72.6 \%$ ) (Fig. $4 \mathrm{~A}$ ) $[18-26,31,32]$. The OS also improved upon addition of an immune checkpoint inhibitor with chemotherapy as the first-line therapy (HR, $-0.30 ; 95 \% \mathrm{CI},-0.45$ to $-0.14 ; \mathrm{I}^{2}=72.2 \%$ ) (Fig. $4 \mathrm{~B}$ ). However, there was no significant difference between patients who received immunotherapy compared to those who took platinum-based chemotherapy in terms of PFS (HR, $-0.24 ; 95 \% \mathrm{CI},-0.61$ to $0.14, \mathrm{I}^{2}=90.4 \%$; Fig. $4 \mathrm{~A}$ ) and $\mathrm{OS}$
(HR, $-0.20 ; 95 \% \mathrm{CI},-0.43$ to $0.03 ; \mathrm{I}^{2}=64.2 \%$; Fig. $\left.4 \mathrm{~B}\right)$ [27-30].

Furthermore, the subgroup analysis of patients with PD-L1 expression less than 1\% revealed that both the PFS and OS were prolonged in the combination of immune therapy and chemotherapy compared with chemotherapy alone (HR, $-0.33 ; 95 \% \mathrm{CI},-0.45$ to -0.22 ; $\mathrm{I}^{2}=36.2 \%$ (Fig 5A) and HR, $-0.27 ; 95 \% \mathrm{CI},-0.44$ to $-0.10 ; \mathrm{I}^{2}=0.0 \%$ (Fig $\left.5 \mathrm{~B}\right)$ ).

\subsection{Methodological quality and sensitivity analyses}

The methodological quality of the eligible studies is shown in Figure 6. All studies were assessed as level A. The sensitivity analyses showed robustness and reliability of our results.

\section{Discussion}

Our meta-analysis aimed to compare the treatment regimes of immunotherapy alone or in combination with chemotherapy with chemotherapy alone in patients with advanced treatment-naive NSCLC.

Based on all the available information extracted from the included trials, we found that combination of immunotherapy and platinum-based chemotherapy as a first-line therapy has a favorable long-term effect. It is important to note that the studies, which compared the combination of chemotherapy and immunotherapy with chemotherapy alone, included all treatment-naive patients regardless of PD-L1 expression on tumor cells. Due to the functional mechanism of the immune checkpoint inhibitor, expression levels of PD-L1 on tumor cells assessed by immunohistochemistry have been regarded as a 
potential responsive biomarker to these agents [14, 35]. The previous study showed that the treatment efficiency in patients with a higher PD-L1 tumor-expression level was significantly better than those with a lower PD-L1 tumor-expression level, when treated by the immune checkpoints inhibitor $[36,37]$. But, the subgroup analysis of this study showed that patients who had low or negative expression of PD-L1( $\leq 1 \%)$ also benefitted from combined immunotherapy and chemotherapy as a first-line treatment in both PFS and OS. Although favorable long-term survival was observed in combined immunotherapy and chemotherapy group, the initial 3-6 month survival curve often overlaps or even crosses [38], which means that the efficacy of immunotherapy combined with chemotherapy in the early stage of treatment may not be superior to chemotherapy[18-26]. The higher rate of toxicity might offset the therapeutic effect of the combination of immunotherapy and chemotherapy at the onset of treatment [38].

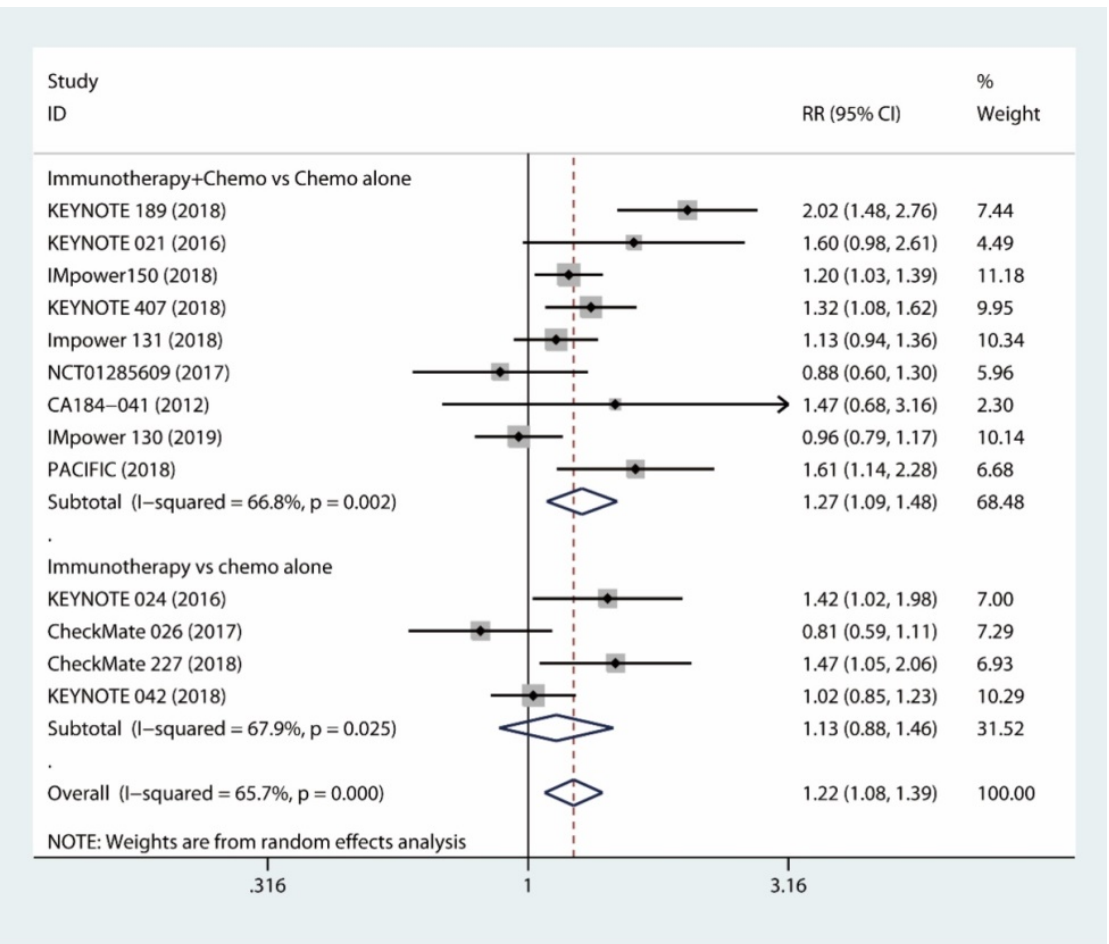

Figure 2. Forest plots of tumor response comparing combination therapy or immunotherapy alone versus chemotherapy alone.

\section{(A) 3-5 grade toxicity}

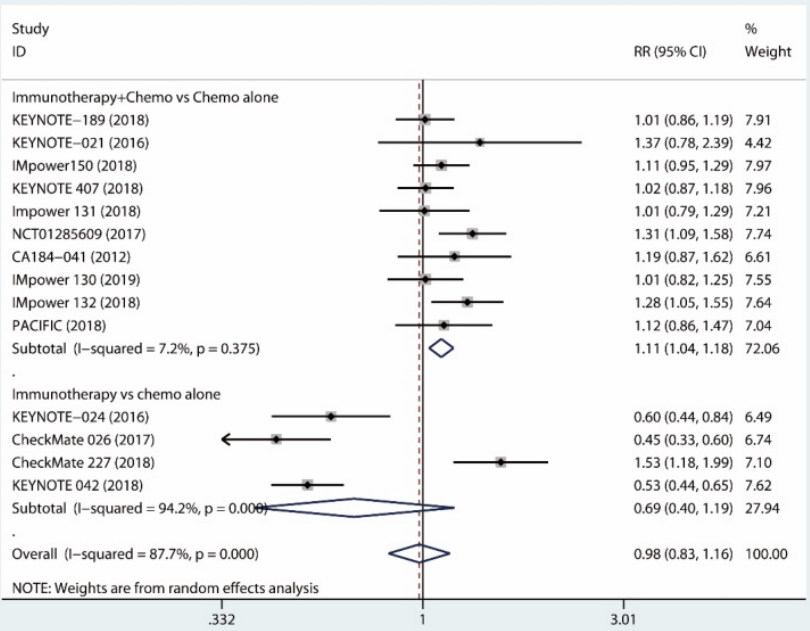

(B) toxicity leading to discontinuation of treatment

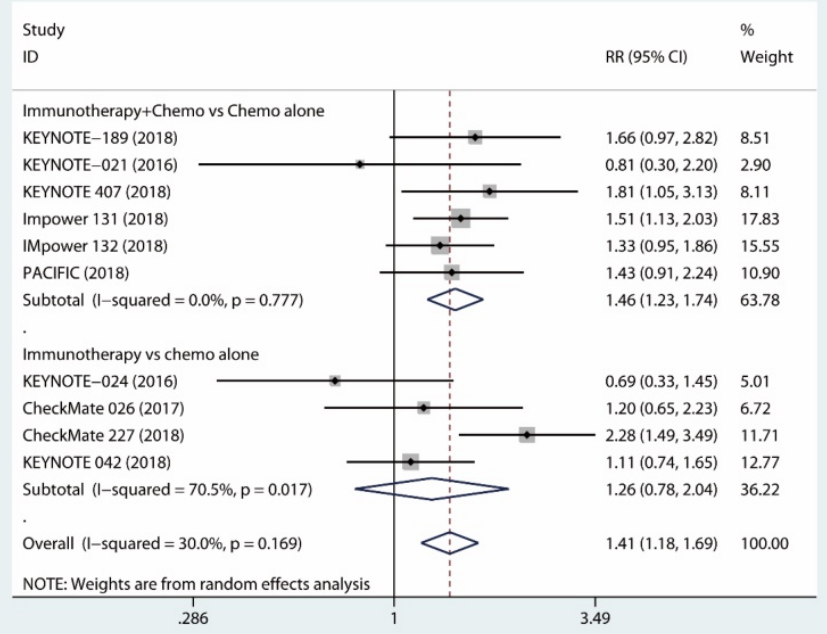

Figure 3. Forest plots of $3-5$ grade toxicity comparing combination therapy or immunotherapy alone versus chemotherapy alone (A). Forest plots of toxicity leading to discontinue of treatment comparing combination therapy or immunotherapy alone versus chemotherapy alone (B). 


\section{(A) PFS of all included patients}

$$
\begin{aligned}
& \text { Study } \\
& \text { ID }
\end{aligned}
$$

Immunotherapy+Chemo vs Chemo alone KEYNOTE 189 (2018)

KEYNOTE 021 (2016)

KEYNOTE 407 (2018)

KEYNOTE 407 (2018)
Impower 131 (2018)

NCT01285609 (2017)

CA184-041 (2012)

IMpower 130 (2019)

IMpower 132 (2018)

PACIFIC (2018)

Subtotal $(1-$ squared $=72.6 \%, \mathrm{p}=0.000)$

Immunotherapy vs chemo alone

KEYNOTE 024 (2016)

CheckMate 026 (2017)

CheckMate 227 (2018)

KEYNOTE 042 (2018)

Subtotal $(1-$ squared $=90.4 \%, p=0.000)$

Overall (I-squared $=87.0 \%, p=0.000)$

NOTE: Weights are from random effects analysis

$$
-1.17
$$

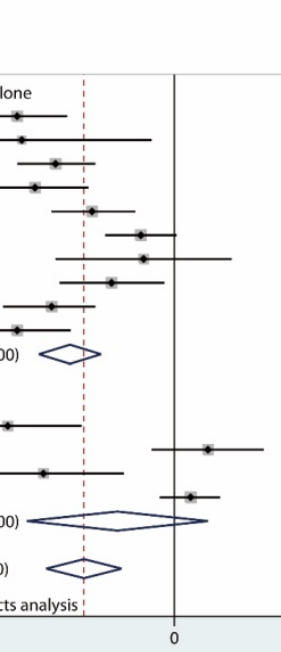

\section{(B) OS of all included patients}

\begin{tabular}{l} 
HR $\left(95 \%\right.$ Cl) $\quad \begin{array}{l}\% \\
\text { Weight }\end{array}$ \\
$-0.65(-0.84,-0.457 .63$ \\
$-0.63(-1.17,-0.094 .30$ \\
$-0.49(-0.65,-0.337 .96$ \\
$-0.58(-0.80,-0.367 .42$ \\
$-0.34(-0.51,-0.167 .86$ \\
$-0.14(-0.29,0.01) 8.07$ \\
$-0.13(-0.49,0.24) 5.90$ \\
$-0.26(-0.48,-0.047 .44$ \\
$-0.51(-0.71,-0.337 .69$ \\
$-0.65(-0.87,-0.437 .45$ \\
$-0.43(-0.56,-0.3171 .73$ \\
$-0.69(-0.99,-0.396 .56$ \\
$0.14(-0.09,0.37) 7.30$ \\
$-0.54(-0.89,-0.216 .17$ \\
$0.07(-0.06,0.19) \quad 8.24$ \\
$-0.24(-0.61,0.14) 28.27$ \\
$-0.38(-0.53,-0.22) 00.00$ \\
\hline 5
\end{tabular}

Study

ID

HR $(95 \% \mathrm{Cl}) \quad$ Weight

Immunotherapy+Chemo vs Chemo alon

KEYNOTE-189 (2018)

KEYNOTE-021 (2016)

IMpower150 (2018)

KEYNOTE 407 (2018)

Impower 131 (2018)

NCT01285609 (2017)

CA184-041 (2012)

IMpower 130 (2019)

IMpower 132 (2018)

IMpower 132 (2018)
PACIFIC (2018)
Subtotal $(\mathrm{I}-$ squared $=72.2 \%, \mathrm{p}=0.000)$

Immunotherapy vs chemo alone

KEYNOTE-024 (2016)

CheckMate 026 (2017)

KEYNOTE 042 (2018)

Subtotal $(1-$ squared $=64.2 \%, p=0.061)$

Overall (l-squared $=69.6 \%, p=0.000)$

NOTE: Weights are from random effects analysis

$-1.17$

$-0.71(-0.97,-0.45) 7.88$ $-0.71(-0.97,-0.45) 7.88$ $-0.25(-0.45,-0.04) 9.15$ $-0.45(-0.71,-0.16) 7.57$ $-0.04(-0.25,0.17) 9.05$ $-0.09(-0.26,0.07) 9.99$ $-0.01(-0.40,0.38) 5.50$ $-0.36(-0.62,-0.09) 7.87$ $-0.21(-0.45,0.03) 8.37$ $-0.30(-0.45,-0.14) 75.60$

$-0.51(-0.89,-0.12) 5.53$ $0.02(-0.22,0.26) \quad 8.26$ $-0.21(-0.34,-0.07) 10.60$ $-0.20(-0.43,0.03) 24.40$

$-0.27(-0.40,-0.15) 100.00$

5

Figure 4. Forest plots of progress free survival comparing combination therapy or immunotherapy alone versus chemotherapy alone (A). Forest plots of overall survival comparing combination therapy or immunotherapy alone versus chemotherapy alone (B).

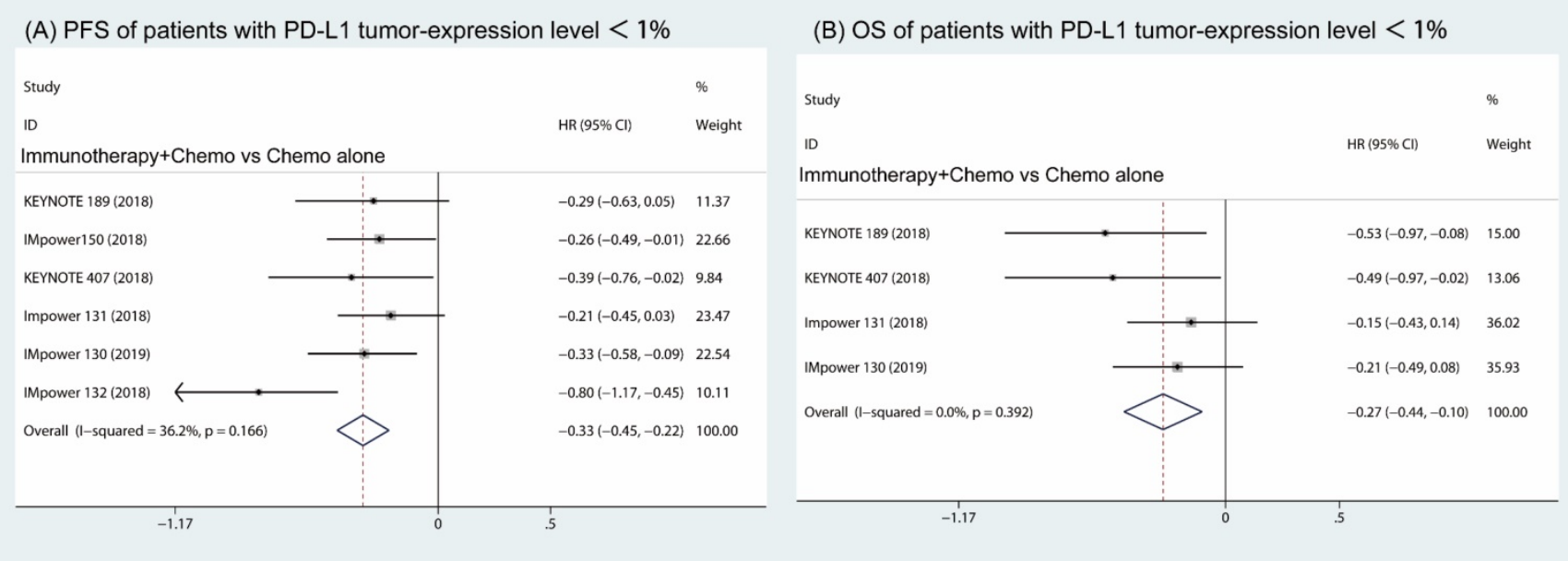

Figure 5. Subgroup analysis of patients with PD-L1 tumor-expression level < $1 \%$ in combination therapy versus chemotherapy alone. Forest plots of progress free survival (A) and overall survival (B).

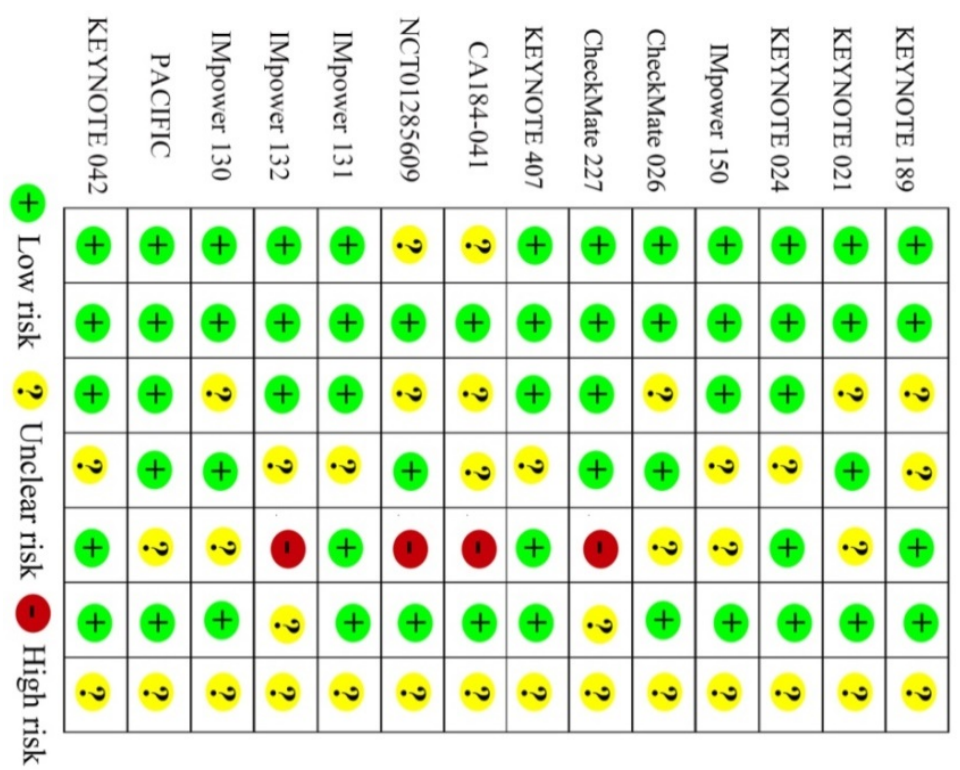

Random sequence generation (selection bias)

Allocation concealment (selection bias)

Blinding of participants and personnel (performance bias)

Blinding of outcome assessment (detection bias)

Incomplete outcome data (attrition bias)

Selective reporting (reporting bias)

Other bias

Figure 6. Risk of bias of the included trials 
Comparison of monotherapy arms of immune-checkpoint inhibitors and chemotherapy shows that selected patients with positive PD-L1 tumors [27, 29, 30] or a high mutation burden [28], who were administered immunotherapy alone, experienced longer PFS and OS. However, the difference was not significant with high heterogeneity among the studies. The results of the KEYNOTE-024 study demonstrated an advantage of pembrolizumab over chemotherapy in patients with a PD-L1 tumor-expression level $\geq 50 \%$ with regards to long-term survival. PFS improvement of 4.3 months was observed in the pembrolizumab group, despite the finding that $43 \%$ of patients who had undergone chemotherapy crossed over to the pembrolizumab group [29]. The results of KEYNOTE-042 [30] which recruited treatment-naïve stage IIIB-IV NSCLC patients with a PD-L1 tumor-expression level $\geq 1 \%$ demonstrated patients in the pembrolizumab group enduring a prolonged OS compared with chemotherapy group. The subgroup analysis of patients with a PD-L1 tumor-expression level $\geq 50 \%$ showed that the PFS and OS were significantly longer in pembrolizumab group compared with chemotherapy group. However, in patients with a PD-L1 tumor-expression level $\langle 50 \%$, there was no significant difference in PFS and OS between the two groups [30]. Based on these observations, we proposed that pembrolizumab alone is effective in patients a PD-L1 tumor-expression level $\geq 50 \%$. However, in the CheckMate 026 study, no significant advantage of nivolumab over chemotherapy was observed in terms of objective tumor response and long-term survival among patients with PD-L1 expression $>5 \%$ [27]. The subgroup analysis demonstrated that even in patients with PD-L1 expression $>50 \%$, there is no significant difference between nivolumab monotherapy group and chemotherapy group. Positive detection of PD-L1 on tumor cells alone may not be sufficient to predict outcomes among patients who receive immunotherapy. Owing to the complexity of the immune system, patients with low- PD-L-1 expression may also benefit from immunotherapy. Thus, new biomarkers, such as tumor mutational burden for response to immune-oncological agent, beyond PD-L1 expression levels, may be the most critical markers for selecting patients for immunotherapy. The CheckMate 026 trial demonstrated that among patients with a high tumor-mutation burden, nivolumab monotherapy achieved a higher response rate compared to chemotherapy alone ( $47 \%$ vs. $28 \%$ ). The median PFS of patients receiving nivolumab monotherapy (9.7 months) has been reported to be much longer than those receiving chemotherapy alone (5.8 months) [27]. Furthermore, the CheckMate 227 trial demonstrated that PFS was significantly longer with first-line nivolumab plus ipilimumab (7.2 months) compared to chemotherapy (5.5 months) among NSCLC patients with a high tumor mutational burden ( $\geq 10$ mutations per megabase), irrespective of PD-L1 expression levels.

Numerous limitations of these trials may hinder the fairness of this meta-analysis. Since only 14 RCTs were included in this meta-analysis, the results were underpowered. Furthermore, the HRs and corresponding 95\% CIs were mainly extracted from the original studies without access to individualized data, which might have contributed to reporting bias. High heterogeneity was observed among the included studies, which may have decreased the strength of our meta-analysis. The high heterogeneity may be explained by the following: First, different immune checkpoint inhibitor regimens were used in different studies. Second, inclusion criteria differed among the included studies. Third, different treatment strategies had been used among studies.

The results of this meta-analysis demonstrated the superiority of combined immunotherapy with chemotherapy over chemotherapy alone in terms of tumor response and long-term survival as a first-line treatment strategy for advanced NSCLC patients but with higher rate of 3 - 5 grade toxicity. And immune-checkpoint inhibitors alone showed similar tumor response, toxicity and long-term outcomes compared to platinum-based chemotherapy in selected patients.

\section{Acknowledgements}

The present study was funded by the Human Provincial Science and Technology Department (2017JJ2345) and the National Natural Science Foundation of China (No. 81673516).

\section{Competing Interests}

The authors have declared that no competing interest exists.

\section{References}

1. Zhou C, Wu Y-L, Chen G, Feng J, Liu X-Q, Wang C, et al. Erlotinib versus chemotherapy as first-line treatment for patients with advanced EGFR mutation-positive non-small-cell lung cancer (OPTIMAL, CTONG-0802): a multicentre, open-label, randomised, phase 3 study. The Lancet Oncology. 2011; 12: 735-42.

2. Siegel RL, Miller KD, Jemal A. Cancer Statistics, 2017. CA Cancer J Clin. 2017; 67: 7-30.

3. Hong QY, Wu GM, Qian GS, Hu CP, Zhou JY, Chen LA, et al. Prevention and management of lung cancer in China. Cancer. 2015; 121 Suppl 17: 3080-8.

4. Chen Y, Peng X, Zhou Y, Xia K, Zhuang W. Comparing the benefits of chemoradiotherapy and chemotherapy for resectable stage III A/N2 non-small cell lung cancer: a meta-analysis. World J Surg Oncol. 2018; 16: 8.

5. Yang JC, Wu YL, Schuler M, Sebastian M, Popat S, Yamamoto N, et al. Afatinib versus cisplatin-based chemotherapy for EGFR mutation-positive lung adenocarcinoma (LUX-Lung 3 and LUX-Lung 6): analysis of overall survival data from two randomised, phase 3 trials. Lancet Oncol. 2015; 16: 141-51. 
6. Xia N, An J, Jiang OQ, Li M, Tan J, Hu CP. Analysis of EGFR, EML4-ALK, KRAS, and c-MET mutations in Chinese lung adenocarcinoma patients. Exp Lung Res. 2013; 39: 328-35.

7. Zhou C, Wu YL, Chen G, Feng J, Liu XQ, Wang C, et al. Final overall survival results from a randomised, phase III study of erlotinib versus chemotherapy as first-line treatment of EGFR mutation-positive advanced non-small-cell lung cancer (OPTIMAL, CTONG-0802). Ann Oncol. 2015; 26: 1877-83.

8. Goldstraw P, Chansky K, Crowley J, Rami-Porta R, Asamura H, Eberhardt WE, et al. The IASLC Lung Cancer Staging Project: Proposals for Revision of the TNM Stage Groupings in the Forthcoming (Eighth) Edition of the TNM Classification for Lung Cancer. J Thorac Oncol. 2016; 11: 39-51.

9. Polanski J, Jankowska-Polanska B, Rosinczuk J, Chabowski M, Szymanska-Chabowska A. Quality of life of patients with lung cancer. Onco Targets Ther. 2016; 9: 1023-8

10. Van Allen EM, Miao D, Schilling B, Shukla SA, Blank C, Zimmer L, et al. Genomic correlates of response to CTLA-4 blockade in metastatic melanoma. Science. 2015; 350: 207-11.

11. Herbst RS, Baas P, Kim D-W, Felip E, Pérez-Gracia JL, Han J-Y, et al. Pembrolizumab versus docetaxel for previously treated, PD-L1-positive, advanced non-small-cell lung cancer (KEYNOTE-010): a randomised controlled trial. The Lancet. 2016; 387: 1540-50.

12. Fehrenbacher L, Spira A, Ballinger M, Kowanetz M, Vansteenkiste J, Mazieres $\mathrm{J}$, et al. Atezolizumab versus docetaxel for patients with previously treated non-small-cell lung cancer (POPLAR): a multicentre, open-label, phase 2 randomised controlled trial. The Lancet. 2016; 387: 1837-46.

13. Lee JS, Lee KH, Cho EK, Kim DW, Kim SW, Kim JH, et al. Nivolumab in advanced non-small-cell lung cancer patients who failed prior platinum-based chemotherapy. Lung Cancer. 2018; 122: 234-42.

14. Borghaei H, Paz-Ares L, Horn L, Spigel DR, Steins M, Ready NE, et al. Nivolumab versus Docetaxel in Advanced Nonsquamous Non-Small-Cell Lung Cancer. New Engl J Med. 2015; 373: 1627-39.

15. Brahmer J, Reckamp KL, Baas P, Crino L, Eberhardt WE, Poddubskaya E, et al. Nivolumab versus Docetaxel in Advanced Squamous-Cell Non-Small-Cell Lung Cancer. New Engl J Med. 2015; 373: 123-35.

16. Bordoni R, Ciardiello F, von Pawel J, Cortinovis D, Karagiannis T, Ballinger M, et al. Patient-Reported Outcomes in OAK: A Phase III Study of Atezolizumab Versus Docetaxel in Advanced Non-Small-cell Lung Cancer. Clin Lung Cancer. 2018.

17. Lazzari C, Bulotta A, Ducceschi M, Viganò MG, Brioschi E, Corti F, et al. Historical evolution of second-line therapy in non-small cell lung cancer. Frontiers in medicine. 2017; 4: 4

18. Gandhi L, Rodriguez-Abreu D, Gadgeel S, Esteban E, Felip E, De Angelis F, et al. Pembrolizumab plus Chemotherapy in Metastatic Non-Small-Cell Lung Cancer. New Engl J Med. 2018; 378: 2078-92.

19. Langer CJ, Gadgeel SM, Borghaei H, Papadimitrakopoulou VA, Patnaik A, Powell SF, et al. Carboplatin and pemetrexed with or without pembrolizumab for advanced, non-squamous non-small-cell lung cancer: a randomised, phase 2 cohort of the open-label KEYNOTE-021 study. The Lancet Oncology. 2016; 17: $1497-508$

20. Socinski MA, Jotte RM, Cappuzzo F, Orlandi F, Stroyakovskiy D, Nogami N, et al. Atezolizumab for First-Line Treatment of Metastatic Nonsquamous NSCLC. New Engl J Med. 2018; 378: 2288-301.

21. Paz-Ares L, Luft A, Vicente D, Tafreshi A, Gumus M, Mazieres J, et al. Pembrolizumab plus Chemotherapy for Squamous Non-Small-Cell Lung Cancer. New Engl J Med. 2018

22. Jotte RM, Cappuzzo F, Vynnychenko I, Stroyakovskiy D, Abreu DR, Hussein MA, et al. IMpower131: Primary PFS and safety analysis of a randomized phase III study of atezolizumab+ carboplatin+ paclitaxel or nab-paclitaxel vs carboplatin+ nab-paclitaxel as 1L therapy in advanced squamous NSCLC. J Clin Oncol. 2018; 36: LBA9000.

23. Papadimitrakopoulou V, Cobo M, Bordoni R, Dubray-Longeras P, Szalai Z, Ursol G, et al. IMPOWER132: PFS and safety results with $1 \mathrm{~L}$ atezolizumab+ carboplatin/cisplatin+ pemetrexed in stage IV non-squamous NSCLC. J Thorac Oncol. 2018; 13.

24. West $\mathrm{H}$, McCleod M, Hussein M, Morabito A, Rittmeyer A, Conter HJ, et al. Atezolizumab in combination with carboplatin plus nab-paclitaxel chemotherapy compared with chemotherapy alone as first-line treatment for metastatic non-squamous non-small-cell lung cancer (IMpower130): a multicentre, randomised, open-label, phase 3 trial. The Lancet Oncology. 2019.

25. Lynch TJ, Bondarenko I, Luft A, Serwatowski P, Barlesi F, Chacko R, et al. Ipilimumab in combination with paclitaxel and carboplatin as first-line treatment in stage IIIB/IV non-small-cell lung cancer: results from a randomized, double-blind, multicenter phase II study. J Clin Oncol. 2012; 30: 2046-54

26. Govindan R, Szczesna A, Ahn M-J, Schneider C-P, Gonzalez Mella PF, Barlesi F, et al. Phase III trial of ipilimumab combined with paclitaxel and carboplatin in advanced squamous non-small-cell lung cancer. J Clin Oncol. 2017; 35: 3449-57.

27. Carbone DP, Reck M, Paz-Ares L Creelan B, Horn L Steins M, et al. First-Line Nivolumab in Stage IV or Recurrent Non-Small-Cell Lung Cancer. New Engl J Med. 2017; 376: 2415-26.

28. Hellmann MD, Ciuleanu TE, Pluzanski A, Lee JS, Otterson GA Audigier-Valette C, et al. Nivolumab plus Ipilimumab in Lung Cancer with a High Tumor Mutational Burden. New Engl J Med. 2018; 378: 2093-104.
29. Reck M, Rodriguez-Abreu D, Robinson AG, Hui R, Csoszi T, Fulop A, et al. Pembrolizumab versus Chemotherapy for PD-L1-Positive Non-Small-Cell Lung Cancer. New Engl J Med. 2016; 375: 1823-33.

30. Mok TSK, Wu YL, Kudaba I, Kowalski DM, Cho BC, Turna HZ, et al. Pembrolizumab versus chemotherapy for previously untreated, PD-L1-expressing, locally advanced or metastatic non-small-cell lung cancer (KEYNOTE-042): a randomised, open-label, controlled, phase 3 trial. Lancet. 2019; 393: 1819-30.

31. Antonia SJ, Villegas A, Daniel D, Vicente D, Murakami S, Hui R, et al. Durvalumab after Chemoradiotherapy in Stage III Non-Small-Cell Lung Cancer. New Engl J Med. 2017; 377: 1919-29.

32. Antonia SJ, Villegas A, Daniel D, Vicente D, Murakami S, Hui R, et al. Overall Survival with Durvalumab after Chemoradiotherapy in Stage III NSCLC. New Engl J Med. 2018; 379: 2342-50.

33. Eisenhauer EA, Therasse P, Bogaerts J, Schwartz LH, Sargent D, Ford R, et al. New response evaluation criteria in solid tumours: revised RECIST guideline (version 1.1). Eur J Cancer. 2009; 45: 228-47.

34. Higgins J. Green S. Cochrane handbook for systematic reviews of interventions Version 5.1. 0. The Cochrane Collaboration. Confidence intervals. 2011.

35. Nishino M, Ramaiya NH, Hatabu H, Hodi FS. Monitoring immune-checkpoint blockade: response evaluation and biomarker development. Nat Rev Clin Oncol. 2017; 14: 655-68.

36. Topalian SL, Hodi FS, Brahmer JR, Gettinger SN, Smith DC, McDermott DF, et al. Safety, activity, and immune correlates of anti-PD-1 antibody in cancer. New Engl J Med. 2012; 366: 2443-54

37. Garon EB, Rizvi NA, Hui R, Leighl N, Balmanoukian AS, Eder JP, et al. Pembrolizumab for the treatment of non-small-cell lung cancer. New Engl J Med. 2015; 372: 2018-28.

38. Champiat S, Ferrara R, Massard C, Besse B, Marabelle A, Soria JC, et al. Hyperprogressive disease: recognizing a novel pattern to improve patient management. Nat Rev Clin Oncol. 2018; 15: 748-62. 